\title{
莫 Fermilab
}

$\overline{\mathrm{p}}$ Note \#399

Bunch-Coalescing RF Cavities at $h=53$

In Main Ring Operationa1 Procedures

Q. ZuBao 
The langitudineh entrance of $\bar{P}$ beam injected into the main Ring is about $1.5 \mathrm{ev}$-sec. But Main Ring is not capable of accelerating such a large emittrince in a single bench Without excessive particle loss at $8 \mathrm{GeV}$ so that the bear u is broken up to several $(i-11)$ adjacent bunches. accelerated to $150 \mathrm{GeV}$ ind reconstituted into a single bunch at $150 \mathrm{GeN}$. Two P.P.A cavities are used for constituting.

The P.P.A cavity is a double cavity and a drift tube injected within it $750 \mathrm{~kg}$ of Ferroxicube 404 are - used to resonate a double cavity over the frequency Range 6 to $30 \mathrm{MHz}$. It is realized by tuning DC basis current. Two. 4CW 10000 tubes work in push -pull to excite the cavity. The bus bars couple two halves cavity together. For theirbeam rerconstitutiongrapplication the tuning buss bar willie removes. This means that tuning $D C$ basis circuit has been broken and one cavity was

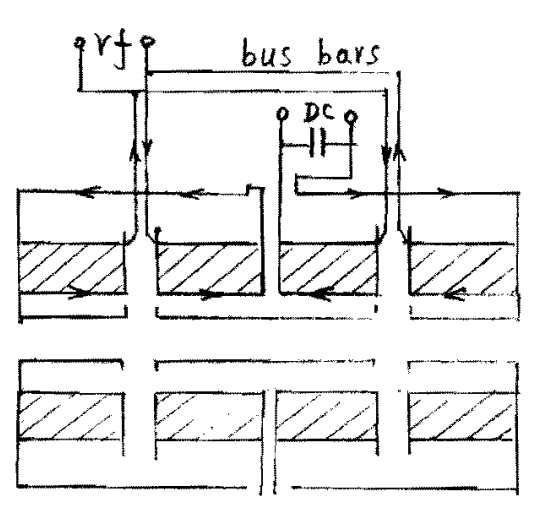
broken up ti two. fortunately. the cavity will only be operating at one frequency. Fig 1 is P.PA cavity in longitudinal section.

Fig. P.P.A Cavity in longitudinal section 
2

In order to constitute $13 \quad h=1113$ bunches into a Single bunch at $150 \mathrm{GeV}$. Using $h=21 \quad(f=1 \mathrm{MHz})$ bucket to manipulate the bean um has suggested, but P.P.A. can not Work at such a low frequency because of magnetic field flux intensity $B$ and power loss too high, so a $h=53$ with some higher havmanice terms added non-sinusoidal RF bucket is used. ale ven $k=1113$ buckets are about $208 \mathrm{~ns}$.

Calculation shows the non-sinusiondal wave has to have about $1.24 \pi$ linear portion in length. According to calculations the peak voltage needed is about $32 \mathrm{kV}$. The Waveform is shows in Fig z (a). The function of $f\left(\omega_{0} t\right)$ is as follow

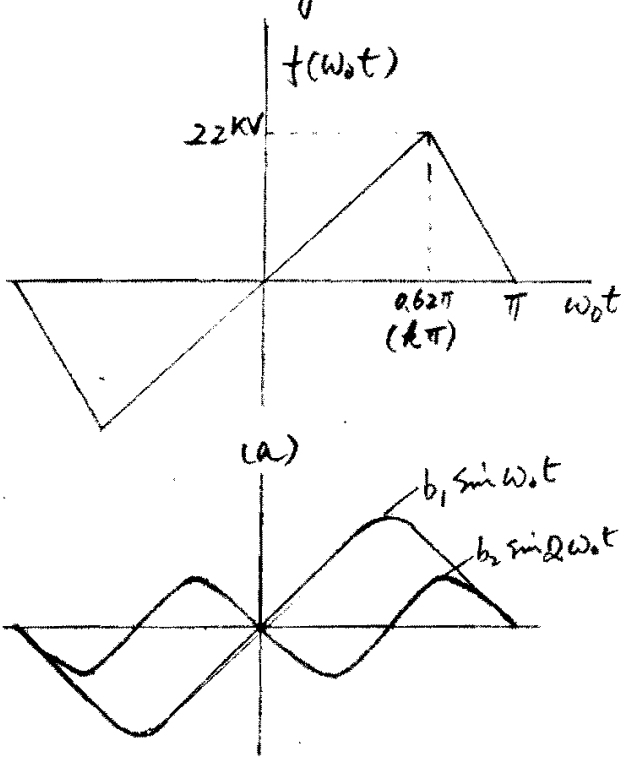

(b)

$$
f\left(\omega_{0} t\right)= \begin{cases}\frac{1}{(1-k) \pi} \omega_{0} t & 0 \leqslant \omega_{0} t \leq(1+k) \pi \\ \frac{1}{k \pi}\left(\pi-\omega_{0} t\right) & (1-k) \pi \leq \omega_{0} t \leq(1+k) \pi \\ \frac{1}{(1-k \pi)}\left(\omega_{0} t-2 \pi\right) & (1+k) \pi \leq \omega_{0} t \leq 2 \pi\end{cases}
$$

Fourier waveform analysis

$$
\begin{aligned}
& f\left(\omega_{0} t\right)=\sum_{n=1}^{\infty} b_{n} \sin n \omega_{\lambda} t \\
& b_{n}=\frac{2}{\pi} \int_{0}^{\pi} f\left(\omega_{0} t\right) \sin n \omega_{0} t d \omega_{0} t=\frac{2 \sin n(1-k) \pi}{n^{2} \pi^{2} k(1-l)} \\
& \text { If } k=0.62 \quad b_{1}=0.80 \quad b_{2}=0.147 \quad b_{3}=0.040 \quad b_{4}=0.054 \\
& V_{\text {peak }}=22 \mathrm{kv} \quad V_{1}=17.6 \quad V_{2}=3.33 \quad V_{3}=0.89 \quad V_{4}=1.18
\end{aligned}
$$

Fig 2 a) requirement Waveform

b) fundamental and second harmonic If $k=07 \quad b_{1}=0.78 \quad b_{2}=0.229 \quad V_{3}=0.033 \quad b_{4}=0.035$ components and relative phase

$V_{\text {peak }}=22 \times v V_{1}=17.17 \quad V_{2}=5.04 \quad V_{3}=0.704 \quad V_{4}=0.77$

It is seen that the fundamental and second harmon are important all other higher harmonic terms only have a. small influence. Fig zees) shows the phase relation of them. 
3

In order to satisfy the requirement of longitudinal manipulating, the P.P.A cavity has to be rebuilt, the input ciraint and output circuit have been modified.

The basic idea for input circuit is to make it as simple as possible and low driving power needed. Since Cavity only operates at a single frequency and tuning $D C$ bis circuit is not used, the one end of input circuit has been grounded, it makes driving and measurement easy. The input resonant circuit simply consists of a air-cored Solenoid and a capacitor. A new transmission transformer has been used. The rate of input voltage to output voltage is $1 / 4$, the rate of infedarice about 1/16. Using this transformer the driving power can greatly reduce at the sane input impedance and it gives a possibility using higher grid basis voltage to reduce conductive angle of tube. consequently to enhance the efficiency. The driving peak Voltage at grid never reached 600". after transformer changed it can reach to about $1000^{\circ}$. As the driving porter and Duty factor $(<3 \%)$ are low. the input terminal load is not necessary. But for reducing $Q$ and making input impedance $50 \Omega$ at resonant frequency. ten $2^{W} 100^{\Omega}$ resistors paroled are put int the resonant circuit. The $Q$ is about 12 . The input circuit shows in Fig 3 .

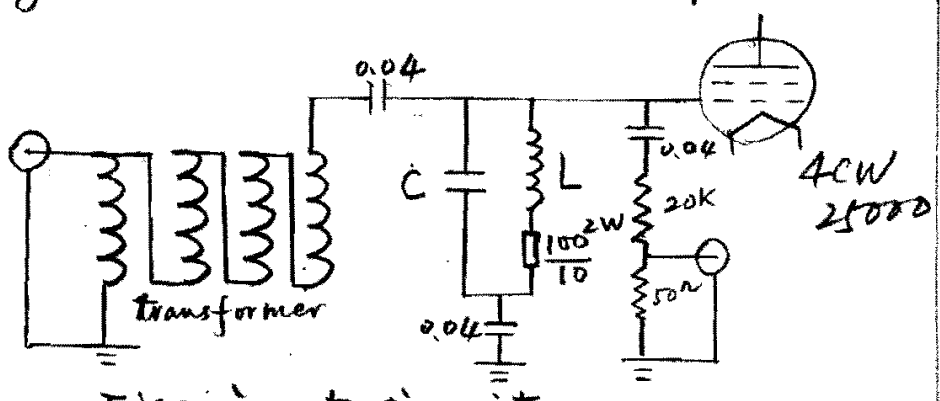

Figs input circuit 
4

Sometime for only one cavity working and higher output power needed in future 4,CW2500 tube has been used.

There are two methods to add higher harmonic teams one is that three half cavities work at the fundminental frequency and one half cavity works at second harmonic, then four gap voltages superimposed. This is simple and eng let it will increase a number of hardware of diving and control. It is difficult to get requirement RF Voltage in only one canty; because the half cavity (one gap) which is operating at fundamental frequency can not get such a high Voltage (about $17,5 \mathrm{KV}$ peak). The other method is that both fundamental anil second harmonic voltage get in one gap (a half cavity). Even though this is difficult, the efficiency is high and it gives a chance the voltage needed gets in one cavity. The latter are described here.

As one knows a pulse of plate current delivered by the tube operating in class $c$ to the output circuit contains Components of the fundamental and mont higher havmanic frequencies. To generate out ant power that is a harmonic of the exciting voltage applied to the control grid. it is merely necessary to resonate the plate circuit to the desired harmonic frequency. If the plate circuit consists of two seriesed circuits which resonates at fundamental frequency and second harmonic frequency respectively, the plate output power will contain two frequency components. The 
6.

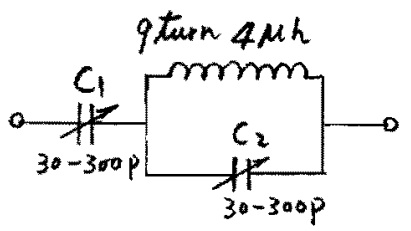

A simple circuit shown in Fig h has been put across the gap of P.P.A cavity to Fig 6. get non-sinusoidal voltage waveform. The ferrite permeability $\mu$ changes with magnetic field flux intensity B. Consequently with plate RF Voltage. For different output power both $c_{1}$ and $c_{2}$ have different Voles to get resonant frequency. The plate ont put equirekant circuit shown in Fig 7. For convenience first

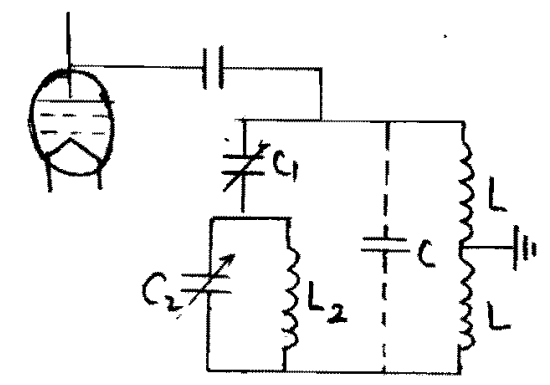

Fig 7 plate out put equivalent circuit C gap capacitor $L$ ferrite inductor
Using imperancemeter set the circuit resonant frequencies nearly harmianic frequencies ("cold condition") then exciting cavity at reguivemest output power condition ("hot conditions) turing $c_{1}$ and $c_{2}$ again to get suitable Waveform. But the suitable phase relation and the ratio of amplitude are not easy to get at the same trine, so If only one gap used, it is difficult to get desired Waveform.

A P.P.A cavity has two gaps, only one gap adds second harmonic component, then two gap - voltages superimpose to get the required ${ }^{+}$voltage. There are two selections to superimpose two sides. One is that both sides resonate at fundamental frequency, at the save time, one of them has complex impedance at second harmonic frequency. In this condition the fundamental wave phase of two sides are the same. The 
7

second harmonic has a phase shift. The amplitude ratio is set by adjusting steven $D C$ bairns Voltage. Sometime for some reason, such as two sides fixative phase shift different, fundamental wave impedance not really resistor, the fundamental phase between two sides is not gro. It can be compensated by adjusting exciting voltage phases. This condition shown in Fig 8 .

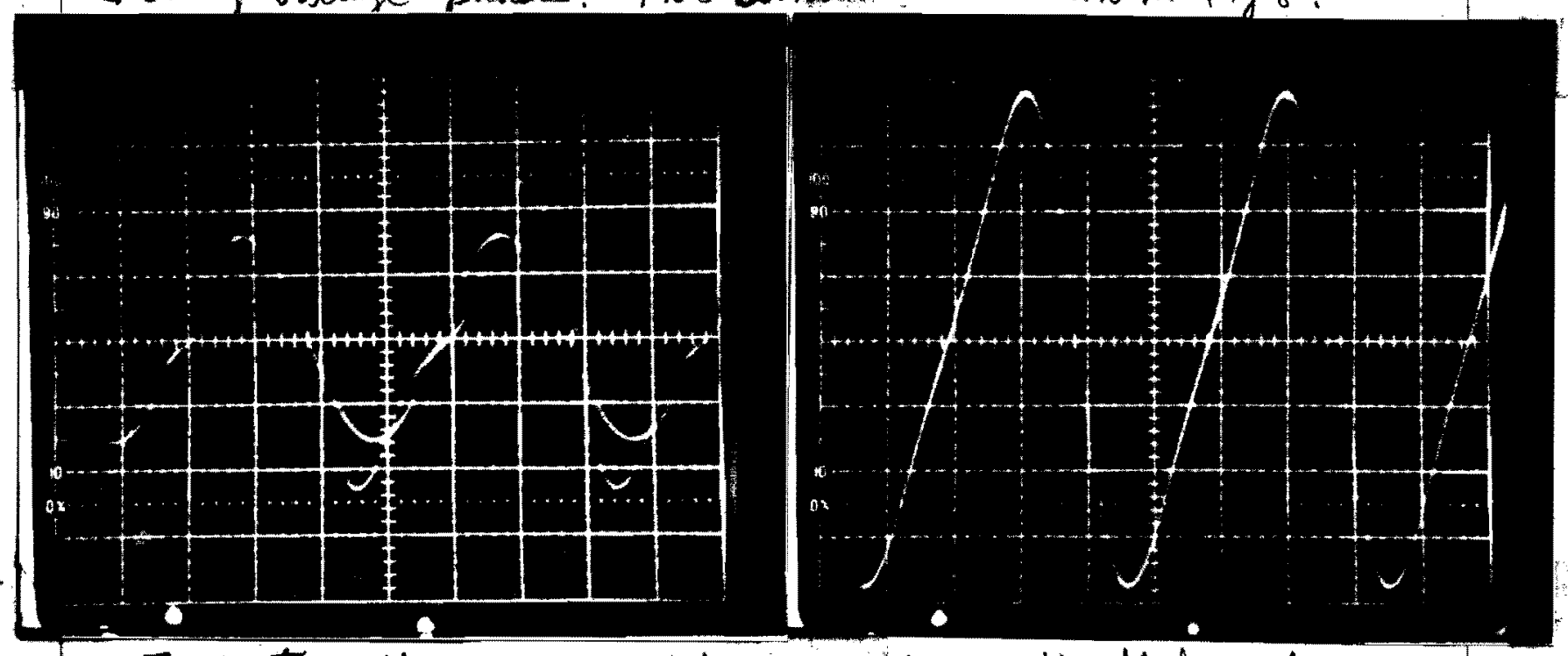

Fig 8. two sides resonance a) two gap waveform b) added waveform The other is that one side resonates at fundamental frequency, other side does not really resonate at any harmamio frequency. The desirable superimposed waveform has been gut by turing $c_{1}$ and $c_{2}$. Never mind what one side voltage Waveform is, as ling as the added Waveform is desired. Waveform as shown in Fig 9

At "hot condition", As second harmonic added in the same gap. The resonant frequency can not be measured, so it is not easy to say what condition it is operating in, only from waveform got some inforrication. Normerally in between two. Here is an example in Fig 10 . The impedance data was measured at "cold condition" by Using impedance meter. The operating frequency 
4.

$$
\text { gturns \&uit }
$$

$4 k$

$$
53-7-13
$$

$$
\frac{\operatorname{cop} p}{11}
$$

8

$\sim$

$\sigma: 1:-1$

$\longrightarrow$

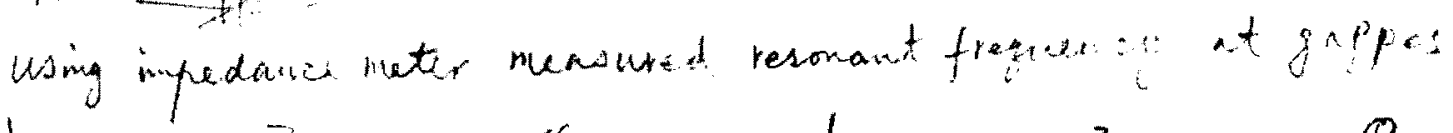

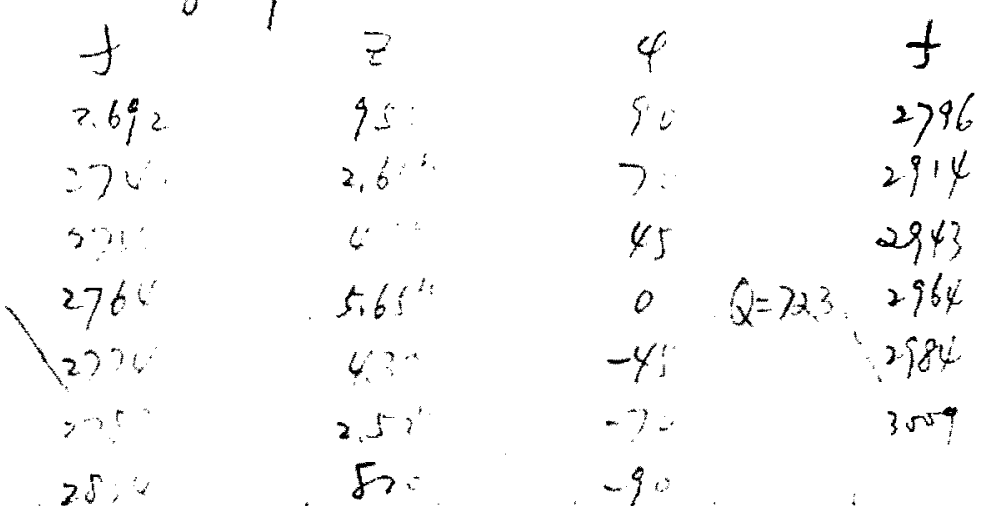

$\begin{array}{cc}z & 9 \\ 80 & 90 \\ 2.81 & 70 \\ 4.8^{k} & 45 \\ 6.6^{k} & 0 \\ 1.2^{\circ} & -45 \\ 3.11 & -70\end{array}$

$$
\begin{aligned}
& V_{p}=8 \mathrm{kV} \\
& V_{s}=400 \mathrm{~V} \\
& V_{g}=-400 \mathrm{~V}
\end{aligned}
$$

$-45$

$-70$

Driving RF Vitty solverk

- is

The phase between two sile is stant $180^{\circ}$
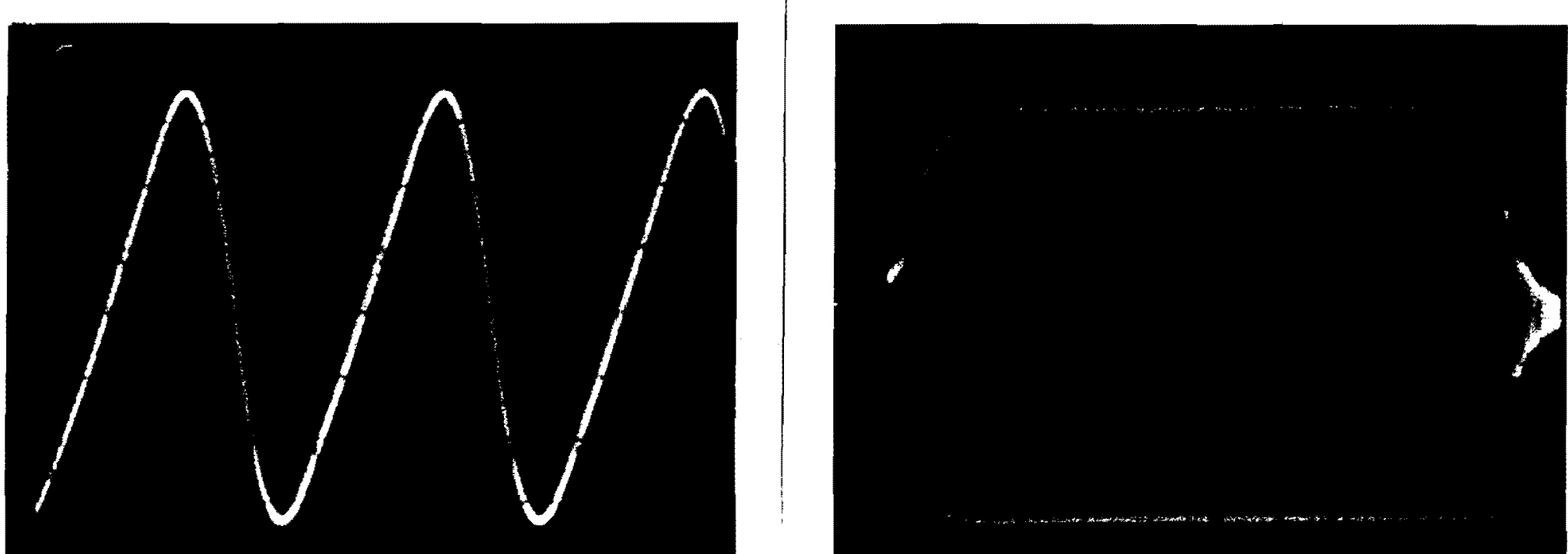

$1 \times 3600 \mathrm{~V} / \mathrm{DIV}$

$0.1 \mathrm{MS} / \mathrm{DN}$

$f=25294 \mathrm{MHZ}$

$\theta=0.64 \pi$
$1 \times 3600^{\circ} / D N$

$20115 / \mathrm{pN}$

Fhepeich à soms

Fig 10 Two sides added non-sinusuidal wavefow example 4 \#P.RA cavity 
9

$\infty$

Fig $q$ one side resonance at fundamental frequency.

a) two gap waveform

b) added waveform.

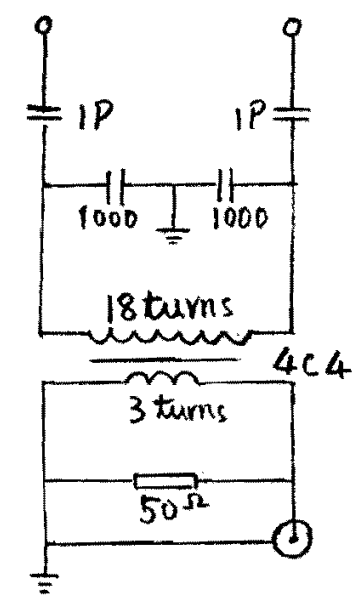

Fig the monitor is $2.5294 \mathrm{MHz}$, output RF Voltage is about $12.5^{\mathrm{kV}}$ peak e. In fact the $c_{1}$ mainly influences the fundamental resonant frequency, the $c_{2}$ mainly influences the second harmonic resonant frequency.

Since all the voltage Waveform and amplitude are got from the monitor the phase shift and amplitude rates of monitor must be the same in two sides. The monitor shown in Fig ll has been used, the ferrite is ferroxembe 4.44.

The conclusion is that for single working frequency using this method to add higher harmonic components can get better linear waveform. high efficiency and less hardware. 
10

The adjustment steps as follow.

(1) Using impedance meter set the output innit resonates nearby harmonic frequencies.

(2) timing $C_{1}$ ti make Voltage amplitude to reach maximum it means the circuit resonates nearby fundamental frequency.

(3) turing $c_{2}$ to make two harmonize components: have suitable phase relation. at this condition the wave form will be fold symmetry.

(4) estimating the amplitude rate of two components. If it is not suitable. tune $c_{1}$ and $c_{2}$ to change the rate but to try keeping suitable phase relation.

After practicing a few times one would know How could do it easier and frater. Maybe step (2)-4) is not necessary just watching and tuning.

(5) two sides added trimming $c_{1}$ and $c_{2}$ to get desirable waveform and amplitude. 